Pacific Journal of Mathematic 


\title{
ON STARLIKENESS AND CONVEXITY OF CERTAIN ANALYTIC FUNCTIONS
}

\author{
V. V. ANH AND P. D. TUAN
}

Let $N$ be the class of normalised regular functions

$$
f(z)=z+\sum_{k=2}^{\infty} a_{k} z^{k}, \quad|z|<1 .
$$

For $0 \leqq \lambda<1, \gamma \geqq 1$, let $f(z), g(z) \in N$ be such that

$$
|f(z) /[\lambda f(z)+(1-\lambda) g(z)]-\gamma|<\gamma, \quad|z|<1 .
$$

We establish the radius of starlikeness of $f(z)$ under the assumption $\operatorname{Re}\{g(z) / z\}>0$, or $\operatorname{Re}\{g(z) / z\}>1 / 2$, or $\operatorname{Re}\left\{z g^{\prime}(z) / g(z)\right\}>$ $\alpha, 0 \leqq \alpha<1$, or $\operatorname{Re}\left\{1+z g^{\prime \prime}(z) / g^{\prime}(z)\right\}>0$ for $|z|<1$. The analysis may be extended to the problem of finding the radius of convexity for certain subclasses of $N$.

1. Introduction and notation. Let $S, S^{*}, S^{c}$ denote the subclasses of $N$ which are univalent, univalent starlike, univalent convex in $|z|<1$ respectively.

A necessary and sufficient condition for $f(z) \in N$ to be univalent starlike in $|z|<r$ is

$$
\operatorname{Re}\left\{\frac{z f^{\prime}(z)}{f(z)}\right\}>0, \quad|z|<r .
$$

A necessary and sufficient condition for $f(z) \in N$ to be univalent convex in $|z|<r$ is

$$
\operatorname{Re}\left\{1+\frac{z f^{\prime \prime}(z)}{f^{\prime}(z)}\right\}>0, \quad|z|<r .
$$

A function $f(z)$ belongs to $S^{*}(\beta)$, i.e., is starlike of order $\beta$, $0 \leqq \beta<1$, if it satisfies the condition

$$
\operatorname{Re}\left\{\frac{z f^{\prime}(z)}{f(z)}\right\}>\beta, \quad|z|<1 .
$$

A function $f(z)$ belongs to $S^{c}(\beta)$, i.e., is convex of order $\beta, 0 \leqq$ $\beta<1$, if it satisfies the condition

$$
\operatorname{Re}\left\{1+\frac{z f^{\prime \prime}(z)}{f^{\prime}(z)}\right\}>\beta, \quad|z|<1 .
$$

Let $\mathscr{P}_{\alpha}$ denote the class of regular functions of the form

$$
p(z)=1+\sum_{k=1}^{\infty} c_{k} z^{k}, \quad|z|<1,
$$


satisfying the inequality $\operatorname{Re}\{p(z)\}>\alpha$ for $|z|<1,0 \leqq \alpha<1$ and $\mathscr{Q}_{r}$ the class of functions $q(z)$ with expansion of the above form but satisfying the inequality $|q(z)-\gamma|<\gamma$ for $|z|<1, \gamma \geqq 1$. We note that both $\mathscr{P}_{0}$ and $\mathscr{Q}_{\infty}$ reduce to the class $\mathscr{P}$ of functions with positive real part.

Let $N_{n}, n \geqq 1$, denote the subclass of $N$ consisting of functions of the form $f(z)=z+\sum_{k=n+1}^{\infty} a_{k} z^{k}$. Then $N_{1}=N$.

Shah [8] considered the problem of determining the radius of starlikeness of $f(z) \in N_{n}$ for the following cases:

(a) $f(z) /[\lambda f(z)+(1-\lambda) g(z)] \in \mathscr{P}$ with $g(z) \in N_{n}$ and $g(z) / z \in \mathscr{P}$, or $g(z) / z \in \mathscr{P}_{1 / 2}\left(\right.$ with $n=1$ ), or $g(z) \in S^{*}(\alpha)$;

(b) $f(z) /[\lambda f(z)+(1-\lambda) g(z)] \in \mathscr{Q}_{1}$ with $g(z) \in N_{n}$ and $g(z) / z \in \mathscr{P}$, or $g(z) \in S^{*}(\alpha)$.

The conditions were shown to be sharp only when $\lambda=0$. In this paper, we solve the problem for the subclasses of $N$ mentioned at the beginning, subject to certain restrictions on the values of $\lambda$. Letting $\gamma \rightarrow \infty$ we obtain the radii of starlikeness of $f(z)$ satisfying $f(z) /[\lambda f(z)+(1-\lambda) g(z)] \in \mathscr{P}$. All the bounds obtained are best possible. Furthermore, the same technique may be used to establish the radius of convexity of $f(z) \in N$ satisfying $f^{\prime}(z) /\left[\lambda f^{\prime}(z)+(1-\lambda) g^{\prime}(z)\right] \in \mathscr{Q}_{r}$, where $g(z)$ belongs to various subclasses of $N$. The results proved here generalize those of MacGregor [3, 4, 5] and Ratti [6, 7].

It should be remarked that parallel results for subclasses of $N_{n}, n>1$, may be derived in an analogous manner. The manipulations involved are, however, more complicated.

The lemmas required for the proofs of our theorems are given in $\S 2$. Section 3 contains theorems giving the conditions for starlikeness. We outline the conditions for convexity in $\S 4$.

2. Some lemmas, Let $\mathscr{B}$ denote the class of functions $w(z)$ regular in $|z|<1$ and satisfying $w(0)=0,|w(z)|<1$ for $|z|<1$.

Lemma 2.1 [9]. If $w(z) \in \mathscr{B}$, then for $|z|<1$,

$$
\left|z w^{\prime}(z)-w(z)\right| \leqq \frac{|z|^{2}-|w(z)|^{2}}{1-|z|^{2}} .
$$

Proof. Write $w(z)=z \phi(z)$, where $\phi(z)$ is regular in $|z|<1$ and $|\phi(z)| \leqq 1$. The assertion now follows from the well-known result due to Caratheodory

$$
\left|\phi^{\prime}(z)\right| \leqq \frac{1-|\phi(z)|^{2}}{1-|z|^{2}}
$$

LEMMA 2.2. Let $w_{1}(z)=[1-w(z)] /[1+\beta w(z)]$, where $w(z) \in \mathscr{B}$, 
$\beta \geqq 0$. Then, fo $|z|=r<\min (1,1 / \beta)$,

$$
\begin{aligned}
\operatorname{Re}\left\{-\beta w_{1}(z)+\frac{1}{w_{1}(z)}\right\} & +\frac{r^{2}\left|1+\beta w_{1}(z)\right|^{2}-\left|1-w_{1}(z)\right|^{2}}{\left(1-r^{2}\right)\left|w_{1}(z)\right|} \\
& \leqq \frac{1-\beta+(3 \beta+1) r+\beta(\beta+3) r^{2}+\beta(\beta-1) r^{3}}{\left(1-r^{2}\right)(1+\beta r)} .
\end{aligned}
$$

Proof. By Schwarz's lemma, $|w(z)| \leqq r$ on $|z|=r<1$. The transformation $w_{1}(z)=[1-w(z)] /[1+\beta w(z)]$ maps the disc $|w(z)| \leqq r$, $r<\min (1,1 / \beta)$, onto the disc $\left|w_{1}(z)-a\right| \leqq d$, where

$$
a=\frac{1-\beta r^{2}}{1-\beta^{2} r^{2}}, \quad d=\frac{(1+\beta) r}{1-\beta^{2} r^{2}} .
$$

Clearly,

$$
0<a-d=\frac{1+r}{1+\beta r}<a+d=\frac{1+r}{1-\beta r} .
$$

Put $w_{1}(z)=a+u+i v, R=|a+u+i v|$; then

$$
\begin{aligned}
S(u, v) & =\operatorname{Re}\left\{-\beta w_{1}(z)+\frac{1}{w_{1}(z)}\right\}+\frac{r^{2}\left|1+\beta w_{1}(z)\right|^{2}-\left|1-w_{1}(z)\right|^{2}}{\left(1-r^{2}\right)\left|w_{1}(z)\right|} \\
& =-\beta(\alpha+u)+\frac{a+u}{R^{2}}+\frac{1-\beta^{2} r^{2}}{1-r^{2}} \cdot \frac{d^{2}-u^{2}-v^{2}}{R} .
\end{aligned}
$$

Now,

$$
\frac{\partial S}{\partial v}=-\frac{v}{R^{4}}\left\{2(a+u)+\frac{1-\beta^{2} r^{2}}{1-r^{2}}\left[\left(d^{2}-u^{2}-v^{2}\right) R+2 R^{3}\right]\right\} .
$$

The terms inside the curly brackets are always positive for $r<$ $\min (1,1 / \beta)$. Hence the maximum of $S(u, v)$ in the disc $\left|w_{1}(z)-a\right| \leqq d$ is attained when $v=0$ and $u \in[-d, d]$. Setting $v=0$ in (2.1) we obtain

$$
S(u, 0)=\frac{2\left(1-\beta^{2} r^{2}\right) a}{1-r^{2}}-\frac{(1+\beta)\left(1-\beta r^{2}\right)}{1-r^{2}}(a+u) .
$$

Since $d S(u, 0) / d u<0$ for $r<\min (1,1 / \beta)$, the maximum of $S(u, 0)$ occurs at the end point $u=-d$ and the result follows.

LEMMA 2.3. If $w(z) \in \mathscr{B}, \beta \geqq 0$, then for $|z|=r<\min (1,1 / \beta)$,

$$
\operatorname{Re}\left\{\frac{z w^{\prime}(z)}{[1-w(z)][1+\beta w(z)]}\right\} \leqq \frac{r}{(1-r)(1+\beta r)} .
$$

Proof. From Lemma 2.1, we have 


$$
\begin{aligned}
\operatorname{Re}\left\{\frac{z w^{\prime}(z)}{(1-w(z))(1+\beta w(z))}\right\} \leqq & \operatorname{Re}\left\{\frac{w(z)}{(1-w(z))(1+\beta w(z))}\right\} \\
& +\frac{r^{2}-|w(z)|^{2}}{\left(1-r^{2}\right)|1-w(z)||1+\beta w(z)|} .
\end{aligned}
$$

Put $w_{1}(z)=[1-w(z)] /[1+\beta w(z)]$, then the above inequality becomes $\operatorname{Re}\left\{\frac{z w^{\prime}(z)}{(1-w(z))(1+\beta w(z))}\right\} \leqq \frac{1}{(1+\beta)^{2}}\left[\beta-1+\operatorname{Re}\left\{-\beta w_{1}(z)+\frac{1}{w_{1}(z)}\right\}\right.$

$$
\left.+\frac{r^{2}\left|1+\beta w_{1}(z)\right|^{2}-\left|1-w_{1}(z)\right|^{2}}{\left(1-r^{2}\right)\left|w_{1}(z)\right|}\right] \text {. }
$$

An application of Lemma 2.2 to the right hand side will give the result which is easily seen to be sharp for $w(z)=z$ at $z=r$.

The following lemma is a consequence of [2, Theorem 3].

LEMma 2.4. If $p(z) \in \mathscr{P}$, then on $|z|=r$,

$$
\begin{aligned}
\operatorname{Re}\left\{\frac{z p^{\prime}(z)}{1+p(z)}\right\} \geqq & \left\{\begin{array}{cll}
-\frac{r}{1+r} \quad, & \text { for } & r<\frac{1}{3} \\
\frac{r^{2}+2^{3 / 2}\left(1-r^{2}\right)^{1 / 2}-3}{1-r^{2}}, & \text { for } \quad \frac{1}{3} \leqq r<1 .
\end{array}\right. \\
& \operatorname{Re}\left\{\frac{z p^{\prime}(z)}{p(z)}\right\} \geqq-\frac{2 r}{1-r^{2}} .
\end{aligned}
$$

\section{Radii of starlikeness.}

THEOREM 3.1. Let $f(z) \in N$ be such that $f(z) /[\lambda f(z)+(1-\lambda) g(z)] \epsilon$ $\mathbb{Q}_{\gamma}$, where $g(z) \in N$ and $g(z) / z \in \mathscr{P}, 0 \leqq \lambda<(1+\sqrt{3}+1 / 2 \gamma) /(2+\sqrt{3})$. Then the radius of starlikeness $\sigma_{2}$ of $f(z)$ is given by the only positive root in $(0,1)$ of the equation

$$
\beta r^{3}+(2+3 \beta) r^{2}+3 r-1=0,
$$

where $\beta=[(1+\lambda) \gamma-1] /(1-\lambda) \gamma$.

Proof. Put $\psi(z)=1-f(z) / \gamma[\lambda f(z)+(1-\lambda) g(z)]$. Then $|\psi(z)|<1$ for $|z|<1$ and $\psi(0)=1-1 / \gamma=A$. Let $w(z)=[\psi(z)-A] /[1-A \psi(z)]$. It is clear that $w(z) \in \mathscr{B}$ and $\psi(z)=[w(z)+A] /[1+A w(z)]$ from which we deduce

$$
\frac{z f^{\prime}(z)}{f(z)}=\frac{z g^{\prime}(z)}{g(z)}-\frac{1+A}{1-\lambda} \cdot \frac{z w^{\prime}(z)}{(1-w(z))(1+\beta w(z))},
$$

$\beta=(A+\lambda) /(1-\lambda)$, provided $1-\lambda(1-w(z)) /(1+A w(z)) \neq 0$. Since $|w(z)| \leqq r$ for $|z|=r$ by Schwarz's lemma, it follows that 


$$
1-\lambda(1-w(z)) /(1+A w(z)) \neq 0
$$

if, in particular, $|z|<1 / \beta$.

Now, as $g(z) / z \in \mathscr{P}$, write $g(z) / z=p(z)$, some $p(z) \in \mathscr{P}$. Then $z g^{\prime}(z) / g(z)=1+z p^{\prime}(z) / p(z)$. An application of (2.5) gives

$$
\operatorname{Re}\left\{\frac{z g^{\prime}(z)}{g(z)}\right\} \geqq \frac{1-2 r-r^{2}}{1-r^{2}},|z|=r<1 .
$$

This result together with (3.1) and (2.3) yield

$$
\operatorname{Re}\left\{\frac{z f^{\prime}(z)}{f(z)}\right\} \geqq \frac{1-3 r-(2+3 \beta) r^{2}-\beta r^{3}}{(1-r)(1+\beta r)} .
$$

For the cubic polynomial

$$
F(r)=\beta r^{3}+(2+3 \beta) r^{2}+3 r-1,
$$

$F(0)<0, F(1)=4+4 \beta>0, F(1 / \beta)=\left(3+6 \beta-\beta^{2}\right) / \beta^{2}$. Thus the equation $F(r)=0$ has exactly one root in $(0,1)$ which is in the range $(0,1 / \beta)$ if $\beta<3+2 \sqrt{3}$, i.e., if $\lambda<(1+\sqrt{3}+1 / 2 \gamma) /(2+\sqrt{3})$.

REMARK 3.1. The theorem is sharp for

$$
f(z)=\frac{1-z}{1+\beta z} \cdot \frac{z(1-z)}{(1+z)} .
$$

When $\lambda=0, f(z)$ is starlike in $|z|<\sqrt{5}-2$ if $\gamma \rightarrow \infty$ and in $|z|<$ $(\sqrt{17}-3) / 4$ if $\gamma=1$ as previously shown by Ratti [6, Theorems 1 and 4].

THEOREM 3.2. Let $f(z) \in N$ be such that $f(z) /[\lambda f(z)+(1-\lambda) g(z)] \epsilon$ $\mathscr{Q}_{r}$, where $g(z) \in N$ and $g(z) / z \in \mathscr{P}_{1 / 2}$. Then the radius of starlikeness of $f(z)$ is

$$
\sigma_{2}=\left\{\begin{array}{cr}
r_{1}, & \text { for } 0 \leqq \lambda \leqq 1 / 2 \gamma, \\
r_{2}=\left[2^{1 / 2}(1+\beta)^{1 / 2}-1\right] /(1+2 \beta), & \text { for } 1 / 2 \gamma<\lambda<(\sqrt{5}+1 \\
& +1 / \gamma) /(\sqrt{5}+3),
\end{array}\right.
$$

where $\beta=[(1+\lambda) \gamma-1] /(1-\lambda) \gamma$ and $r_{1}$ is the smallest positive root in $(0,1)$ of the equation

$$
\begin{aligned}
\left(1+2 \beta+9 \beta^{2}\right) r^{4} & +2\left(1+12 \beta+3 \beta^{2}\right) r^{3}+\left(13+10 \beta+\beta^{2}\right) r^{2} \\
& +4(1-\beta) r-4=0 .
\end{aligned}
$$

Proof. Since $g(z) / z \in \mathscr{P}_{1 / 2}$, there exists $p(z) \in \mathscr{P}$ so that $g(z) / z=$ $1 / 2+p(z) / 2$. Hence 


$$
\frac{z g^{\prime}(z)}{g(z)}=1+\frac{z p^{\prime}(z)}{1+p(z)}
$$

Applying (2.4) to this equation gives, on $|z|=r$,

(3.4) $\quad \operatorname{Re}\left\{\frac{z g^{\prime}(z)}{g(z)}\right\} \geqq\left\{\begin{array}{cl}1 /(1+r), & \text { for } \quad 0<r<1 / 3 \\ 2\left[2^{1 / 2}\left(1-r^{2}\right)^{1 / 2}-1\right] /\left(1-r^{2}\right), & \text { for } 1 / 3 \leqq r<1 .\end{array}\right.$

This result together with (3.1) and (2.3) yield, for $|z|=r<1 / 3$,

$$
\operatorname{Re}\left\{\frac{z f^{\prime}(z)}{f(z)}\right\} \geqq \frac{1-2 r-(1+2 \beta) r^{2}}{(1-r)(1+\beta r)}=G(r)
$$

and for $1 / 3 \leqq r<1$,

$$
\operatorname{Re}\left\{\frac{z f^{\prime}(z)}{f(z)}\right\} \geqq-\frac{(1+\beta) r}{(1-r)(1+\beta r)}+\frac{2\left[2^{1 / 2}\left(1-r^{2}\right)^{1 / 2}-1\right]}{1-r^{2}},
$$

which yields the equation giving the condition of starlikeness of $f(z)$ to be

$$
\begin{aligned}
\left(1+2 \beta+9 \beta^{2}\right) r^{4} & +2\left(1+12 \beta+3 \beta^{2}\right) r^{3}+\left(13+10 \beta+\beta^{2}\right) r^{2} \\
& +4(1-\beta) r-4=0 .
\end{aligned}
$$

The only root in $(0,1)$ of the numerator of $G(r)$ is $r_{2}$ which is less than $1 / 3$ if $\beta>1$, i.e., if $\lambda>1 / 2 \gamma$, and is the range $(0,1 / \beta)$ if $\beta<$ $\sqrt{5}+2$, i.e., if $\lambda<(\sqrt{5}+1+1 / \gamma) /(\sqrt{5}+3)$. Thus $f(z)$ is starlike in $|z|<r_{2}$ if $1 / 2 \gamma<\lambda<(\sqrt{5}+1+1 / \gamma) /(\sqrt{5}+3)$. Now, for $0 \leqq \lambda \leqq$ $1 / 2 \gamma, \beta<1$, and $r_{1}$ is in the interval $(0,1 / \beta)$ and the theorem is proved.

REMARK 3.2. The results are sharp. The extremal functions are

$$
f(z)=\left\{\begin{array}{l}
\frac{1-z}{1+\beta z} \cdot \frac{z}{2}\left[1+\frac{1}{2}\left(\frac{1+z e^{-i \theta}}{1-z e^{-i \theta}}+\frac{1+z e^{i \theta}}{1-z e^{i \theta}}\right)\right\}, \text { for } 0 \leqq \lambda \leqq 1 / 2 \gamma \\
\frac{1-z}{1+\beta z} \cdot \frac{z}{1+z}, \text { for } 1 / 2 \gamma<\lambda<(\sqrt{5}+1+1 / \gamma)(\sqrt{5}+3),
\end{array}\right.
$$

where $\theta$ satisfies the equation

$$
\begin{aligned}
H\left(r_{1}\right)\left(1+r_{1}^{2}\right) & +r_{1}^{2}-\left[3 H\left(r_{1}\right)+1 / 2+r_{1}^{2}\left(H\left(r_{1}\right)+1 / 2\right)\right] r_{1} \cos \theta \\
& +2 H\left(r_{1}\right) r_{1}^{2} \cos ^{2} \theta=0
\end{aligned}
$$

with

$$
H\left(r_{1}\right)=\left[r_{1}^{2}+2^{3 / 2}\left(1-r_{1}^{2}\right)^{1 / 2}-3\right] / 2\left(1-r_{1}^{2}\right) .
$$

When $\lambda=0$, the cases $\gamma \rightarrow \infty$ and $\gamma=1$ give Theorems 2 and 5 of [6]. 
REMARK 3.3. For $g(z) \in S^{c}$, the result [10]

$$
\operatorname{Re}\left\{\frac{z g^{\prime}(z)}{g(z)}\right\} \geqq \frac{1}{1+r}, \quad|z|=r<1
$$

together with (3.1) and (2.3) give the radius of starlikeness of $f(z) \epsilon$ $N$ with $f(z) /[\lambda f(z)+(1-\lambda) g(z)] \in \mathscr{Q}_{r}$ to be $\left[2^{1 / 2}(1+\beta)^{1 / 2}-1\right] /(1+2 \beta)$ for $0 \leqq \lambda<(\sqrt{5}+1+1 / \gamma) /(\sqrt{5}+3), \beta=[(1+\lambda) \gamma-1] /(1-\lambda) \gamma$. The bound is attained for the function

$$
f(z)=\frac{1-z}{1+\beta z} \cdot \frac{z}{1+z}
$$

When $\lambda=0$, the cases $\gamma \rightarrow \infty$ and $\gamma=1$ become Theorem 4 of [4] and Theorem 4 of [5] respectively.

THEOREM 3.3. Let $f(z) \in N$ be such that $f(z) /[\lambda f(z)+(1-\lambda) g(z)] \epsilon$ $\mathscr{Q}_{\gamma}$, where $g(z) \in S^{*}(\alpha), 0 \leqq \lambda<\lambda_{0}$, some $\lambda_{0}<1$. Then the radius of starlikeness $\sigma_{3}$ of $f(z)$ is given by the smallest positive root in $(0,1)$ of the equation

$$
\beta(2 \alpha-1) r^{3}+(3 \beta+2 \alpha-2 \alpha \beta) r^{2}+(3-2 \alpha) r-1=0,
$$

where $\beta=[(1+\lambda) \gamma-1] /(1-\lambda) \gamma$.

Proof. Since $g(z) \in S^{*}(\alpha)$, we have

$$
\operatorname{Re}\left\{\frac{z g^{\prime}(z)}{g(z)}\right\} \geqq \frac{1+(2 \alpha-1) r}{1+r},|z|=r<1 .
$$

Applying this result and (2.3) to (3.1) gives the required equation from which $\sigma_{3}$ may be obtained. $\lambda_{0}$ is determined by the condition $\sigma_{3}<1 / \beta$.

REMARK 3.4. The theorem is sharp for

$$
f(z)=\frac{1-z}{1+\beta z} \cdot \frac{z}{(1+z)^{2-2 \alpha}} .
$$

When $\lambda=0$, the cases $\gamma \rightarrow \infty$ and $\gamma=1$ correspond to Theorems 3 and 6 of [6].

4. Radii of convexity. In this section, we briefly look at the problem of determining the radius of convexity of $f(z) \in N$ with $f^{\prime}(z) /\left[\lambda f^{\prime}(z)+(1-\lambda) g^{\prime}(z)\right] \in \mathbb{Q}_{r}$, where $g(z)$ belongs to various subclasses of $N$. For such $f(z)$, we can deduce in a similar manner as in Theorem 3.1 that 


$$
\begin{aligned}
\operatorname{Re}\left\{1+\frac{z f^{\prime \prime}(z)}{f^{\prime}(z)}\right\}= & \operatorname{Re}\left\{1+\frac{z g^{\prime \prime}(z)}{g^{\prime}(z)}\right\}-\frac{1+A}{1-\lambda} \\
& \cdot \frac{z w^{\prime}(z)}{(1-w(z))(1+\beta w(z))},
\end{aligned}
$$

provided $1-\lambda(1-w(z)) /(1+A w(z)) \neq 0, w(z) \in \mathscr{B}, A=1-1 / \gamma, \beta=$ $(A+\lambda) /(1-\lambda)$. With some restriction on $\lambda$, we may apply (2.3) and the known bounds for $\operatorname{Re}\left\{1+z g^{\prime \prime}(z) / g^{\prime}(z)\right\}$ to (4.1) to get the equations from which the radii of convexity of $f(z)$ may be obtained. We consider the following six cases.

(i) $g^{\prime}(z) \in \mathscr{P}$. The radius of convexity of $f(z)$ is equal to $\sigma_{1}$ as given by Theorem 3.1.

(ii) $g^{\prime}(z) \in \mathscr{P}_{1 / 2}$. The radius of convexity of $f(z)$ is equal to $\sigma_{2}$ as given by Theorem 3.2.

(iii) $g(z) \in S^{c}(\alpha)$. The radius of convexity of $f(z)$ is equal to $\sigma_{3}$ as given by Theorem 3.3.

(iv) $g(z) \in S$.

The result $[1$, p. 166$]$

$$
\operatorname{Re}\left\{1+\frac{z g^{\prime \prime}(z)}{g^{\prime}(z)}\right\} \geqq \frac{1-4 r+r^{2}}{1-r^{2}},|z|=r<1,
$$

together with (2.3) and (4.1) yield the radius of convexity of $f(z)$ to be the smallest positive root (less than 1 ) of the equation

$$
\beta r^{3}-5 \beta r^{2}-5 r+1=0,
$$

with $0 \leqq \lambda<(2-\sqrt{6}+1 / 2 \gamma) /(3-\sqrt{6})$.

(v) $g(z) \in S^{*}$. The radius of convexity of $f(z)$ is the same as that of part (iv).

(vi) $g(z) \in S^{*}(1 / 2)$. Theorem 4.1 of [9] with $\beta=1 / 2$ gives

$$
\operatorname{Re}\left\{1+\frac{z g^{\prime \prime}(z)}{g^{\prime}(z)}\right\} \geqq \frac{1-r}{1+r}, \quad|z|=r<1 / 2 .
$$

This result together with (2.3) and (4.1) yield the radius of convexity of $f(z)$ to be the smallest positive root $\rho$ of the equation

$$
\beta r^{3}-3 \beta r^{2}-3 r+1=0,
$$

with $0 \leqq \lambda<(1+\sqrt{2}+1 / 2 \gamma) /(2+\sqrt{2})$.

All these results are best possible and generalise those obtained by Ratti [7, Theorems 1-6].

\section{REFERENCES}

1. G. M. Goluzin, Geometric Theory of Functions of a Complex Variable, Amer. Math. Soc., Providence, R. I., 1969. 
2. W. Janowski, Some extremal problems for certain families of analytic functions I, Ann. Polon. Math., 28 (1973), 298-326.

3. T. H. MacGregor, Functions whose derivative has a positive real part, Trans. Amer. Math. Soc., 104 (1962), 532-537.

4. The radius of univalence of certain analytic functions, Proc. Amer. Math. Soc., 14 (1963), 514-520

5. - The radius of univalence of certain analytic functions II, Proc. Amer. Math. Soc., 14 (1963), 521-524.

6. J. S. Ratti, The radius of univalence of certain analytic functions, Math. Z., 107 (1968), 241-248.

7. - The radius of convexity of certain analytic functions, Indian J. Pure Appl. Math., 1 (1970), 30-36.

8. G. M. Shah, On the univalence of some analytic functions, Pacific J. Math., 43 (1972), 239-250.

9. V. Singh and R. M. Goel, On radii of convexity and starlikeness of some classes of functions, J. Math. Soc. Japan, 23 (1971), 323-339.

10. E. Strohhäcker, Beiträge zur Theorie der schlichten Funktionen, Math. Z., 37 (1933), 356-380.

Received October 15, 1976. One of the authors (V. V. Anh) acknowledges the financial support of a University of Tasmania Research studentship. The authors are grateful for the referee's valuable comments.

University of Tasmania, Hobart Tasmania, Australia 



\section{PACIFIC JOURNAL OF MATHEMATICS}

EDITORS

RICHARD ARENS (Managing Editor)

University of California

Los Angeles, California 90024

R. A. Beaumont

University of Washington

Seattle, Washington 98105
J. DUGUNDJI

Department of Mathematics

University of Southern Californı

Los Angeles, California 90007

D. Gilbarg and J. Milgram

Stanford University

Stanford, California 94305

\section{ASSOCIATE EDITORS}

E. F. BECKENBACH

B. H. NeumanN

F. WOLF

K. YoSHIDA

\section{SUPPORTING INSTITUTIONS}

UNIVERSITY OF BRITISH COLUMBIA

CALIFORNIA INSTITUTE OF TECHNOLOGY

UNIVERSITY OF CALIFORNIA

MONTANA STATE UNIVERSITY

UNIVERSITY OF NEVADA

NEW MEXICO STATE UNIVERSITY

OREGON STATE UNIVERSITY

UNIVERSITY OF OREGON

OSAKA UNIVERSITY
UNIVERSITY OF SOUTHERN CALIFORNIA

STANFORD UNIVERSITY

UNIVERSITY OF TOKYO

UNIVERSITY OF UTAH

WASHINGTON STATE UNIVERSITY

UNIVERSITY OF WASHINGTON

AMERICAN MATHEMATICAL SOCIETY

NAVAL WEAPONS CENTER 


\section{Pacific Journal of Mathematics}

Vol. 69 , No. 1

May, 1977

V. V. Anh and P. D. Tuan, On starlikeness and convexity of certain analytic

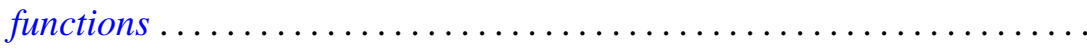

Willard Ellis Baxter and L. A. Casciotti, Rings with involution and the prime

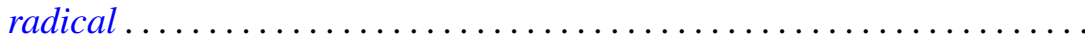

Manuel Phillip Berriozabal, Hon-Fei Lai and Dix Hayes Pettey,

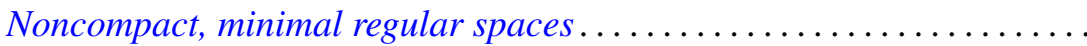

Sun Man Chang, Measures with continuous image law ................

John Benjamin Friedlander, Certain hypotheses concerning

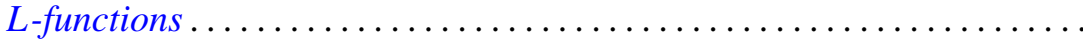

Moshe Goldberg and Ernst Gabor Straus, On characterizations and

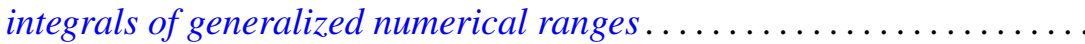

Pierre A. Grillet, On subdirectly irreducible commutative semigroups ...... 55

Robert E. Hartwig and Jiang Luh, On finite regular rings ..............

Roger Hugh Hunter, Fred Richman and Elbert A. Walker, Finite direct sums of cyclic valuated p-groups ........................... 97

Atsushi Inoue, On a class of unbounded operator algebras. III ......... 105

Wells Johnson and Kevin J. Mitchell, Symmetries for sums of the Legendre

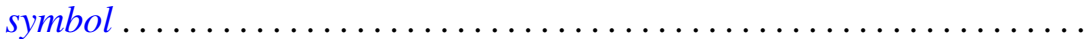

Jimmie Don Lawson, John Robie Liukkonen and Michael William Mislove,

Measure algebras of semilattices with finite breadth

Glenn Richard Luecke, A note on spectral continuity and on spectral properties of essentially $G_{1}$ operators ...............

Takahiko Nakazi, Invariant subspaces of weak-* Dirichlet algebras . .

James William Pendergrass, Calculations of the Schur group ...

Carl Pomerance, On composite $n$ for which $\varphi(n) \mid n-1$. II. . .

Marc Aristide Rieffel and Alfons Van Daele, A bounded operator approach to Tomita-Takesaki theory........................

Daniel Byron Shapiro, Spaces of similarities. IV. $(s, t)$-families ...

Leon M. Simon, Equations of mean curvature type in 2 independent variables.

Joseph Nicholas Simone, Metric components of continuous images of ordered compacta ............................

William Charles Waterhouse, Pairs of symmetric bilinear forms in

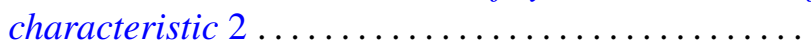

